PROCEEDINGS OF THE

AMERICAN MATHEMATICAL SOCIETY

Volume 134, Number 1, Pages 81-91

S 0002-9939(05)08117-7

Article electronically published on June 14, 2005

\title{
A NOTE ON THE STABILITY OF LOCAL ZETA FUNCTIONS
}

\author{
DIMITER VASSILEV \\ (Communicated by Andreas Seeger)
}

\begin{abstract}
We show the existence of an interval of stability under small perturbations of local zeta functions corresponding to non-trivial local solutions of an elliptic equation with Lipschitz coefficients.

RÉsumé. Nous démontrons l'existence d'un intervalle de stabilité pour la fonction zêta associée à une équation uniformément elliptique du second ordre à coefficients lipschitziens.
\end{abstract}

\section{INTRODUCTION AND STATEMENT OF THE RESULTS}

Let $u$ be a function defined and continuous in the ball $B_{2 R}$ of radius $2 R$ centered at the origin of $\mathbb{R}^{N}$. For $0<2 r<R$ and $\lambda \in \mathbb{C}$ consider the integral

$$
J_{u}(\lambda)=\int_{B_{r}}|u(x)|^{-\lambda} d V,
$$

where $d V$ is the Lebesgue measure on $\mathbb{R}^{N}$. For a fixed $u$ the above integral converges absolutely when the real part $\Re(\lambda)$ of $\lambda$ is negative and defines a holomorphic function of $\lambda$ in this region. A natural question is to find spaces of functions for which $J_{u}(\lambda)$ has a holomorphic extension to the right of the imaginary axis for any $u$ in the considered space. A second question is to ask if the extension is uniform with respect to "small" perturbations of $u$. In other words we are asking for classes of functions for which

$$
t_{o}(u)=\sup \left\{t=\Re(\lambda) \mid J_{u}(\lambda)<\infty\right\}
$$

is strictly positive and furthermore for any $t<t_{o}(u)$ there exists $\epsilon>0$, such that, for every function $v$ in the considered class of functions and satisfying

$$
\sup _{x \in B_{2 R}}|u(x)-v(x)|<\epsilon,
$$

we have $t_{o}(v) \geq t_{o}(u)$.

If we consider the class of functions that are analytic near the origin, it is a classical result that $J_{u}(\lambda)$ can be extended to the whole complex plane as a meromorphic function with poles on the positive real axis; see $[\mathrm{BG}, \mathrm{A}, \mathrm{Be}, \mathrm{Ka}, \mathrm{M}]$ and $[\mathrm{Bj}]$. Having this we are led to study the so-called multiplicity, i.e., the value of the smallest pole. It is clear that the stability to which we referred in the last paragraph is a question about the multiplicity. It is important to have in mind Varchenko's example [V] according to which if $N \geq 3$ we do not have stability in

Received by the editors September 2, 2004.

2000 Mathematics Subject Classification. Primary 11S40.

(C)2005 American Mathematical Society Reverts to public domain 28 years from publication 
the class of real analytic functions. For sharp results in the setting or real analytic functions on $\mathbb{R}^{N}$ or holomorphic functions on $\mathbb{C}^{N}$ we refer the reader to $[\mathrm{DK}],[\mathrm{N}]$, [PSS, [PS1] and [PS2].

Now consider the space of all local solutions of a fixed uniformly elliptic equation with Lipschitz continuous coefficients $a_{i j}$,

$$
\mathcal{L} u=\sum_{i, j=1}^{N} \frac{\partial}{\partial x_{i}}\left(a_{i j}(x) \frac{\partial u}{\partial x_{j}}\right)=0 .
$$

The main result of this note is the following theorem.

Theorem 1.1. Let $L$ be a uniformly elliptic operator with Lipschitz coefficients as in (1.3) and $u$ a non-trivial solution of $\mathcal{L} u=0$ on $B_{2 R}$. There exist positive numbers $R_{o}$ and $t_{u}$, such that, for any $t<t_{u}$ there exists an $\epsilon>0$ with the following property: for every solution $v$ of $\mathcal{L} v=0$ on $B_{2 R}$ satisfying

$$
\sup _{x \in B_{2 R_{o}}}|u(x)-v(x)|<\epsilon
$$

we have $I_{t}(v) \stackrel{\text { def }}{=} \int_{B}|v(x)|^{-2 t} d V<\infty$ for any ball $B, B \subset 2 B \subset R_{o}$, i.e., the local integrability is an open condition for t's in the interval $t<t_{u}$.

As a consequence we can define the multiplicity for any solution $u$ of the considered elliptic equation by setting

$$
t_{o}=\sup \left\{t \mid I_{t}(u)<\infty\right\} .
$$

The above theorem shows that $t_{o}>0$ and there is an interval around $t=0$ on which we have stability under small perturbations. Our proof yields a precise lower bound on $t_{u}$; see (2.16). The very interesting questions of determining the value of $t_{o}$ or showing stability on the whole interval $t<t_{o}$ are not addressed in this note.

We shall give the proof of Theorem 1.1 at the end of Section 2. In Section 3 we make a comment on the stability in the class of holomorphic functions.

\section{2. $A_{p}$ Weights AND THE STABILITY FOR LOCAL ZETA FUnCTIONS}

The key point in the proof of Theorem 1.1 is that every solution of an elliptic equation is an $A_{p}$ weight for a suitable $p>0$ (cf. [GL1, GL2] and see also [GG]) and furthermore the $A_{p}$ norm is controlled by the so-called frequency function. It is well known that a reverse Hölder inequality implies the $A_{p}$ property for some $p$; $\mathrm{CF}$ and $\mathrm{St2}$. In our case a reverse Hölder inequality will hold for the function $u^{2}$ and all $s>1$ (see Theorem 2.4) and our task is to write down the consequences of this fact. For ease of exposition and in order to keep track of the dependence on the various parameters we shall state all the results that lead to the $A_{p}$ property of solutions to elliptic equations in divergence form with Lipschitz coefficients. It is worth comparing our result with the case of polynomial functions, namely for any polynomial $P$ on $\mathbb{R}^{N}$ the function $\log |P|$ belongs to $\mathrm{BMO}\left(\mathbb{R}^{N}\right)$ (cf. St1]), hence the finiteness of the integral $I_{t}(P)$ for some $t>0$. Moreover (see [RSt]) $|P|$ satisfies a reverse Hölder inequality and $|P|^{a}$ is an $A_{p}$ weight if $-1<a d<p-1, p>0$, where $d$ is the degree of the polynomial $P$. However the $A_{p}$ norm depends on the degree $d$ while in our case we have control of the $A_{p}$ norm by the frequency of the function $u$. 
Turning to our problem, let $\mathcal{L}$ be a second-order elliptic operator of the type

$$
\mathcal{L} u=\sum_{i, j=1}^{N} \frac{\partial}{\partial x_{i}}\left(a_{i j}(x) \frac{\partial u}{\partial x_{j}}\right) .
$$

We shall assume $A=\left(a_{i j}(x)\right), i, j=1, \ldots, N$, is a Lipschitz, symmetric and uniformly elliptic matrix. Thus $a_{i j}=a_{j i}$ and there exist $\lambda, \Lambda>0$ such that for all $x, y, \eta \in \mathbb{R}^{N}$ and $i, j=1, \ldots, N$ we have

$$
\lambda|\eta|^{2} \leq<A(x) \eta, \eta>\leq \lambda^{-1}|\eta|^{2} \quad\left|a_{i j}(x)-a_{i j}(y)\right|<\Lambda|x-y| .
$$

Let $B_{R}(x)$ denote the ball with center $x$ and radius $R$, and let $\rho$ be the distance to its center. Suppose $u$ is a weak solution of $\mathcal{L} u=0$ in some ball $B_{R}(x)$. For $r<R$ we define, correspondingly, the height $H_{u}(r)$ and the Dirichlet (energy) integral $D_{u}(r)$ of $u$ on the ball $B_{r}(x)$ by the following formulas:

$$
\begin{gathered}
H_{x, u}(r)=\int_{\partial B_{r}(x)} u^{2}\langle A \nabla \rho, \nabla \rho\rangle d \sigma \\
D_{x, u}(r)=\int_{B_{r}(x)}\langle A \nabla u, \nabla u\rangle d V .
\end{gathered}
$$

We shall use the notation $H(r)$ and $D(r)$ when there is no ambiguity about the function and point we are using. The following lemma provides the key properties of the height and energy associated to a fixed solution $u$. We refer the reader to [GL1, [K] or [GV] for the proof.

Lemma 2.1. Given a linear uniformly elliptic operator $\mathcal{L}$ as above, there exists a constant $M=M(\lambda, \Lambda, N)$ such that for every solution $\mathcal{L} u=0$ in $B_{R}(x)$ the height and energy functions of $u$ satisfy the following inequalities for a.e. $r \in(0, R)$ :

$$
\left|H^{\prime}(r)-\frac{N-1}{r} H(r)-2 D(r)\right| \leq M H(r)
$$

and

$$
D^{\prime}(r) \geq 2 \int_{\partial B_{r}(x)} \frac{\langle A \nabla u, \nabla \rho\rangle^{2}}{\langle A \nabla \rho, \nabla \rho\rangle} d V+\frac{N-2}{r} D(r)-M D(r) .
$$

Note that the inequality for the height function shows that $H^{\prime}(r)>0$ for $0<$ $r<\frac{N-1}{M}$ when $H(r)$ is defined. It will be convenient then to define

$$
R_{o}=\min \left\{\frac{R}{8}, \frac{N-1}{M}\right\},
$$

and hence $H^{\prime}(r)>0$ for $0<r \leq R_{o}$. Therefore, if $u$ is a non-trivial solution (i.e. $u \neq \equiv 0)$, then $H(r) \neq 0$ on $\left(0, R_{o}\right]$. We remark that if $\mathcal{L}$ is the laplacian, we have $M=0$ and the height is an increasing function of $r$ wherever it is defined.

From now on we shall consider only non-trivial solutions. For every ball $B_{r}(x)$ contained in the domain on which we are given a non-trivial solution, $u$ we define the frequency function by

$$
F_{x, u}(r) \stackrel{\text { def }}{=} \frac{r D_{x, u}(r)}{H_{x, u}(r)} e^{2 M r} .
$$

Lemma 2.1 and the above observations imply the following monotonicity theorem. 
Theorem 2.2 (GL1]). Suppose $u$ is a non-trivial solution of $\mathcal{L} u=0$ in $B_{R}(x)$. Under these conditions the frequency $F_{x, u}(r)$ is a continuous monotone non-decreasing function of $r \in\left(0, R_{o}\right]$, with $R_{o}=\min \left\{\frac{R}{8}, \frac{N-1}{M}\right\}$.

With the help of the monotonicity one proves that the measure $u^{2} d V$ is a doubling measure.

Theorem 2.3 (GL1). Suppose $u$ is a weak non-trivial solution of (2.1) in $B_{R}(x)$. With

$$
\tilde{C_{o}}=\lambda^{2} 2^{N} e^{M} 2^{2 F_{x, u}\left(R_{o}\right)},
$$

we have

for all $0<2 r \leq R_{o}$.

$$
\int_{B_{2 r}(x)} u^{2} d V \leq \tilde{C}_{o} \int_{B_{r}(x)} u^{2} d V
$$

The doubling property implies the following reverse Hölder inequalities.

Theorem 2.4 (GL1]). Suppose $u$ is a weak non-trivial solution of (2.1) in $B_{R}(x)$ and let $w=u^{2}$. There exists a positive constant $\mu=\mu(\lambda, \Lambda, N)$ such that with

$$
\tilde{C}_{1}=\frac{\mu \tilde{C}_{o}}{2^{N}}
$$

we have for all $s>1$ and $0<2 r \leq R_{o}$,

$$
\left(\frac{1}{\left|B_{r}(x)\right|} \int_{B_{r}(x)} w^{s} d V\right)^{1 / s} \leq \tilde{C}_{1} \frac{1}{\left|B_{r}(x)\right|} \int_{B_{r}(x)} w d V .
$$

The constant $\mu$ is the constant that appears in the classical Moser estimate bounding the maximum of $u^{2}$ on a ball by the average of $u^{2}$ on a twice as big ball. In the case of laplacian $\mu=2^{N}$ can be seen by the mean value property of harmonic functions.

Remark 2.5. A similar result holds for $w=|\nabla u|^{2}$ for some $s>1$.

Let us denote by $B_{R_{o}}$ the ball centered at the origin with radius $R_{o}$, i.e., $B_{R_{o}}=$ $B_{R_{o}}(0)$. Given a non-trivial solution $u$ on some ball $B_{2 R}$, let $F_{u}$ be the constant defined by

$$
F_{u}=\max \left\{F_{x, u}\left(R_{o}\right) \mid x \in B_{R_{o}}\right\},
$$

where $R_{o}$ was defined in (2.3). By the monotonicity property $F_{u}$ bounds the frequencies on all balls $B$ such that $2 B \subset B_{R_{o}}$, where for a ball $B$ we denote by $2 B$ the ball with the same center as $B$ and twice its radius. It is clear that if $u$ is a non-trivial solution we have that the doubling property of Theorem 2.3 and the reverse Hölder inequality of Theorem 2.4 hold for all balls $B \subset 2 B \subset B_{R_{o}}$ with the same constants $C_{o}$ and $C_{1}$, replacing correspondingly $\tilde{C}_{o}$ and $\tilde{C}_{1}$, and defined respectively by

$$
C_{o}=\lambda^{2} 2^{N} e^{M} 2^{2 F_{u}}
$$

and

$$
C_{1}=\lambda^{2} \mu e^{M} 2^{2 F_{u}} .
$$

Let us also observe that since we have the reverse Hölder inequality for all $s>1$ and $C_{1}$ is independent of $s$, we can let $s \rightarrow \infty$ and obtain the maximum of $w$ on 
the ball. Alternatively, we can directly use Theorem 2.3 and Moser's estimate. In either case, the conclusion is the following theorem.

Theorem 2.6. Suppose $u$ is a weak non-trivial solution of (2.1) in $B_{2 R}$, for some $R>0$. Let $M=M(\lambda, \Lambda, N)$ be the constant from Lemma 2.1, $R_{o}=\min \left\{\frac{R}{8}, \frac{N-1}{M}\right\}$ and $w=u^{2}$. For every ball $B \subset 2 B \subset B_{R_{o}}$ the following inequality holds:

$$
\sup _{B} w \leq C_{1} \frac{1}{|B|} \int_{B} w d V
$$

where $C_{1}$ is defined in (2.8).

It is well known that a reverse Hölder inequality for some $s>1$ implies that $w=u^{2}$ is an $A_{p}$ weight for a suitable $p$ depending on $s$. The Muckenhoupt weight class $A_{p}\left(B_{R_{o}}\right)$ consists of all non-negative measurable functions $w$ for which

$$
\|w\|_{A_{p}} \stackrel{\text { def }}{=} \sup \left[\frac{1}{|B|} \int_{B} w(x) d V\right]\left[\frac{1}{|B|} \int_{B} w(x)^{1-p^{\prime}} d V\right]^{p-1}<\infty,
$$

where the sup is over all balls $B$ with $2 B \subset B_{R_{o}}$.

In our case we have (2.9), so we are going to look for an estimate on the possible $p$ 's. Let us define

$$
p_{o}=\inf \left\{p>1 \mid w \in A_{p}\right\} .
$$

In the following theorem we show that

$$
p_{o} \leq \frac{1+\frac{1}{m} \log _{2} \sigma_{N}}{\frac{1}{m} \log _{2} \sigma_{N}},
$$

where $\sigma_{N}$ denotes the area of the unit sphere in $\mathbb{R}^{N}$. The number $m$, related to the doubling constant for dyadic cubes, is defined by

$$
2^{m}=C_{o}^{2},
$$

where $C_{o}$ is defined in (2.7). As is customary we denote $w(Q)=\int_{Q} w d V$ and for $s \geq 1$ we define $s^{\prime}$ to be the conjugate exponent $\frac{1}{s}+\frac{1}{s^{\prime}}=1$.

Theorem 2.7. If $u$ is a non-trivial solution of $L u=0$ in $B_{2 R}$, then $u^{2} \in A_{p}\left(B_{R_{o}}\right)$ for every $p=\bar{s}^{\prime}$ satisfying

$$
\bar{s}-1<\frac{1}{m} \log _{2} \sigma_{N},
$$

where $m$ is the doubling exponent from (2.11) and $\sigma_{N}$ is the area of the unit sphere in $\mathbb{R}^{N}$.

The fact that $w$ is an $A_{p}$ weight for some $p$ was proven in Theorem 1.1 in [GL1]. Our task here is to prove the explicit bound on $p$, which gives a precise version of the result in GL1. In the proof we will use the following lemma which is a simple corollary from Theorem 2.6 .

Lemma 2.8. Suppose $w \in L_{l o c}^{1}(\Omega), \Omega$ a domain, is a non-negative function such that (2.9) holds true for every ball $B \subset 2 B \subset \Omega$.

a) For every $0<\gamma<1$ and $\delta$ given by

$$
\delta=1-\left(\frac{1-\gamma}{C_{1}}\right) \quad(\Rightarrow 0<\delta<1),
$$


we have the following property: whenever $E \subset B$ and $w(E) \leq \gamma w(B)$ it must be

$$
|E| \leq \delta|B| \text {. }
$$

b) For every $\gamma, 0<\gamma<1$, and $\delta$ given by

$$
\delta=\frac{1}{\sigma_{N}}-\frac{1}{\sigma_{N}}\left(\frac{1-\gamma}{C_{1}}\right) \quad(\Rightarrow 0<\delta<1),
$$

we have the following property: whenever $E \subset Q$ and $w(E) \leq \gamma w(Q)$, it must be

$$
|E| \leq \delta|Q|,
$$

where $Q$ is any cube such that $4 Q \subset \Omega$.

We note that $4 Q$ stays for the cube with the same center as $Q$ and four times its size. With the help of the above lemma we can prove our main theorem.

Proof of Theorem 2.7. By the usual invariances it is enough to prove the claim for $w$ being a measure on a unit dyadic cube $Q_{o}$ satisfying

$$
w\left(Q_{o}\right)=\left|Q_{o}\right|=1 .
$$

Let $m$ be such that $2^{m}=C_{o}^{2}$. By Theorem 2.3 it follows that for any dyadic cube $Q$ and $\tilde{Q}$ a dyadic parent of $Q$,

$$
w(\tilde{Q}) \leq 2^{m} w(Q) .
$$

Define

$$
f(x)=\frac{1}{w(x)} \chi_{Q_{o}}(x)
$$

and the corresponding dyadic maximal function

$$
M f(x)=\sup _{x \in Q} \frac{1}{|Q|} \int_{Q} f w,
$$

where the sup is taken over all dyadic cubes containing $x$. Now we have

$$
\begin{aligned}
\int_{Q_{o}} w^{1-\bar{s}} d V & =\int_{Q_{o}} f^{\bar{s}-1} d V \leq \int_{Q_{o}}(M f)^{\bar{s}-1} d V \\
& \leq \int_{Q_{o} \cap\{M f \leq 1\}}(M f)^{\bar{s}-1} d V+\sum_{k=0}^{\infty} \int_{E^{k} \backslash E^{k+1}}(M f)^{\bar{s}-1} d V,
\end{aligned}
$$

where we define

$$
E^{k}=\left\{x \in Q_{o} \mid M f(x)>2^{n k}\right\}
$$

with $n$ a fixed constant, to be chosen. The first integral in the above sum is bounded by one, so we have to estimate the integrals in the sum.

For this we use the Calderon-Zygmund decomposition, i.e., given $\alpha>0$ we decompose the set $\{M f>\alpha\}$ as a disjoint union of dyadic cubes $\cup Q_{j}$, such that,

$$
\alpha<\frac{1}{\left|Q_{j}\right|} \int_{Q_{j}} f w d V \leq 2^{m} \alpha .
$$

Furthermore, if $\alpha_{1}>\alpha_{2}$, each dyadic cube in the decomposition at level $\alpha_{1}$ is contained in a dyadic cube in the decomposition at level $\alpha_{2}$. 
Going back to the estimate of each of the terms in the sum, let us decompose each of the sets $E^{k}$ using, correspondingly, $\alpha=2^{n k}$ as described above. Therefore we have

$$
\int_{E^{k} \backslash E^{k+1}}(M f)^{\bar{s}-1} d V \leq 2^{n(k+1)(\bar{s}-1)} \int_{E^{k} \backslash E^{k+1}} d V \leq 2^{n(k+1)(s-1)}\left|E^{k}\right| .
$$

Note that

$$
w\left(E^{k} \cap Q\right) \leq 2^{m-n} w(Q),
$$

for every cube $Q$ in the decomposition of $E^{k-1}$. Indeed, by the properties of the decomposition we have

$$
w\left(E^{k} \cap Q\right)=\sum_{Q_{j}} w\left(Q_{j}\right),
$$

where the sum is over all cubes $Q_{j}$ making $E^{k}$ and such that $Q_{j} \subset Q$. Using (2.13) we obtain

$$
\begin{aligned}
w\left(E^{k} \cap Q\right)=\sum_{Q_{j}} w\left(Q_{j}\right) & \leq \frac{1}{2^{n k}} \int_{Q_{j}} f w d V \leq \frac{1}{2^{n k}} \int_{Q} f w d V \\
& \leq \frac{1}{2^{n k}} 2^{m} 2^{n(k-1)} w(Q) \quad\left(Q \text { is of height } E^{k-1} !\right) \\
& \leq 2^{m-n} w(Q) .
\end{aligned}
$$

At this point we note that for any $n>m$ the above inequality combined with Lemma 2.8 gives

$$
\left|E^{k}\right| \leq \delta|Q|
$$

where

$$
\delta=\frac{1}{\sigma_{N}}\left[1-\left(\frac{1-2^{m-n}}{C_{1}}\right)\right], \quad n>m .
$$

Since the cubes of $E^{k-1}$ are contained in the cubes of $E^{k}$, we obtain by summing over the cubes of $E^{k-1}$

$$
\left|E^{k}\right| \leq \delta\left|E^{k-1}\right| \quad \Rightarrow \quad\left|E^{k}\right| \leq \delta^{k}
$$

Putting the estimates together we prove

$$
\int_{Q_{o}} w^{1-\bar{s}} \leq 1+\frac{1}{\delta} \sum_{k=0}^{\infty}\left(2^{n(\bar{s}-1)} \delta\right)^{k+1}=1+\frac{2^{n(\bar{s}-1)}}{1-\delta 2^{n(\bar{s}-1)}}<\infty,
$$

provided $2^{n(\bar{s}-1)} \delta<1$, i.e., $\bar{s}-1<\frac{1}{n} \log _{2} \delta^{-1}$. Let us consider the function $\log _{2} \delta^{-1 / n}$ on the interval $(m, \infty)$, where $\delta=\delta(n)$ is the function in (2.14). Then clearly the integral (2.15) is finite if

$$
\bar{s}-1<\sup _{n>m} \log _{2} \delta^{-1 / n}=\log _{2} \sup _{n \geq m} \delta^{-1 / n} .
$$

Since $\delta(m)=\sigma_{N}^{-1}$ it follows that the integral in (2.15) is finite for any $\bar{s}$ such that

$$
\bar{s}-1<\left.\log _{2} \delta^{-1 / n}\right|_{n=m}=\frac{1}{m} \log _{2} \sigma_{N} .
$$

This concludes the proof of the theorem. 
We are ready to prove Theorem 1.1 .

Proof of Theorem 1.1. Let $u$ be a fixed non-trivial solution of $\mathcal{L} u=0$ in $B_{2 R}$. Let $R_{o}$ be the constant defined in Theorem 2.2. Define the stability multiplicity of $u$ by

$$
t_{u}=\frac{1}{m} \log _{2} \sigma_{N} .
$$

From Theorem 2.7 for $t<t_{u}$ we have $I_{t}(u)<\infty$ on any ball $B$ with $2 B \subset B_{R_{o}}$, hence the first claim of our theorem.

To prove the stability result, it will be enough to show

$$
\left|F_{u}-F_{v}\right|=\left|\sup _{x \in B_{R_{o}}} F_{x, u}\left(R_{o}\right)-\sup _{x \in B_{R_{o}}} F_{x, v}\left(R_{o}\right)\right| \leq C \sup _{B_{2 R_{o}}}|u-v| .
$$

Indeed, assuming the above inequality and taking into account that $m=2 \log _{2} C_{o}$ depends continuously on the quantity $F_{u}$ (see (2.7) and (2.6) ) it follows that there exists an $\epsilon>0$, such that, if $\sup _{B_{2 R_{o}}}|u-v|<\epsilon$ and $t<t_{u}$, then $t_{u}-t>\left|t_{u}-t_{v}\right|$, and hence $t<t_{v}$.

At this point we are left with proving (2.17) to which task we turn. Let $x \in B_{R_{o}}$ and let $v$ be any solution of $\mathcal{L} v=0$ satisfying

$$
\sup _{B_{2 R_{o}}}|u-v|<\frac{1}{2} \sup _{B_{2 R_{o}}}|u|
$$

We have

$$
\begin{gathered}
\left|F_{x, u}\left(R_{o}\right)-F_{x, v}\left(R_{o}\right)\right| \leq \frac{R_{o} e^{2 M R_{o}}}{H_{x, u}\left(R_{o}\right) H_{x, v}\left(R_{o}\right)}\left[D_{x, u}\left(R_{o}\right)\left|H_{x, u}\left(R_{o}\right)-H_{x, u}\left(R_{o}\right)\right|\right. \\
\left.+H_{x, u}(r)\left|D_{x, u}\left(R_{o}\right)-D_{x, u}\left(R_{o}\right)\right|\right] .
\end{gathered}
$$

For $v$ as above and any $y \in B_{R_{o}}(x)$ the triangle inequality implies

$$
\left|u^{2}(y)-v^{2}(y)\right| \leq \frac{5}{2} \sup _{B_{2 R_{o}}}|u| .
$$

Hence the height of $v$ is bounded by the height of $u$ as follows:

$$
\left|H_{x, u}\left(R_{o}\right)-H_{y, v}\left(R_{o}\right)\right| \leq \frac{3}{2} \lambda N R_{o}{ }^{N-1} \sigma_{N} \sup _{B_{2 R_{o}}}|u| \sup _{B_{R_{o}}}|u-v| .
$$

Furthermore, let us observe that $h_{o}=\inf _{x \in B_{R_{o}}} H_{x, u}\left(R_{o}\right)$ is a positive constant, $h_{o}>0$, since $u$ is a non-trivial solution. On the other hand, Caccioppoli's inequality yields for any $x \in B_{R_{o}}$ the inequality

$$
\left|D_{x, u}\left(R_{o}\right)-D_{y, v}\left(R_{o}\right)\right| \leq C \sup _{B_{2 R_{o}}}|u-v|,
$$

with a constant $C$ depending on $\Lambda, \lambda, R_{o}, N$ and $\sup _{B_{2} R_{o}}|u|$. Putting the above estimates together we come to

$$
\left|F_{x, u}\left(R_{o}\right)-F_{x, v}\left(R_{o}\right)\right| \leq C \sup _{B_{2 R_{o}}}|u-v|,
$$

with a constant $C=C\left(\lambda, \Lambda, N, \sup _{B_{2} R_{o}}|u|, \inf _{x \in B_{R_{o}}} \int_{\partial B_{R_{o}}(x)} u^{2} d \sigma\right)$, which concludes the proof of the theorem. 


\section{LOCAL ZETA FUNCTIONS FOR HOLOMORPHIC FUNCTIONS}

Let us now consider integrals of the type

$$
I_{t}(f)=\int_{B_{r}}|f(z)|^{-2 t} d V
$$

where $B_{R} \subset \mathbb{C}^{N}$ is the ball of radius $R$ centered at the origin and $f$ is holomorphic in $B_{2 R}$. Using the convexity of $u \mapsto u^{-t}, u \in \mathbb{R}$, and the fact that the real and imaginary parts of $f$ are harmonic, we immediately obtain the following theorem.

Theorem 3.1. Let $f$ be a not identically vanishing holomorphic function on $B_{2} R_{o}$ and let $0<2 r<R_{o}$. There exists a $t(f)>0$ such that for any $t<t(f)$ there exists an $\epsilon>0$ such that for every $g$ holomorphic in $B_{2 R_{o}}$ and satisfying

$$
\sup _{z \in B_{2 r}}|f(z)-g(z)|<\epsilon
$$

we have $I_{t}(g)<\infty$.

As pointed out by the referee, the above theorem can also be proved by invoking the Weierstrass Preparation theorem with parameters, as formulated for example in PSS.

It is an interesting question if Theorem 1.1 in the case of $L$ being the laplacian is in fact identical to the above theorem. We recall the following fact (see Proposition 6.2.2 of $[\mathrm{Av}]$ ) which provides a link between the two classes of functions.

Theorem 3.2. Let $h$ be function harmonic in the ball $B_{R} \subset \mathbb{R}^{n}$ and for $0<r<R$ let $\tilde{h}$ be its holomorphic extension in the Lie ball $U_{r} \subset \mathbb{C}^{N}$. If

$$
m(r)=\max \{|h(x)|:|x|=r\}
$$

and

$$
M\left(r^{\prime}\right)=\max \left\{|\tilde{h}(z)|:|z|=r^{\prime}\right\}, \quad r^{\prime}<\frac{r}{\sqrt{2}}<\frac{R}{\sqrt{2}}
$$

then

$$
M\left(r^{\prime}\right) \leq 3 \cdot 2^{\frac{N}{2}-1}\left(1-2 \frac{{r^{\prime}}^{2}}{r^{2}}\right)^{-\frac{N}{2}} m(r)
$$

Recall that the Lie ball is defined by

$$
U_{r}=\left\{z \in \mathbb{C}^{N}:\left(|z|^{2}+\left(|z|^{4}-\left|\sum_{j=1}^{N} z_{j}^{2}\right|^{2}\right)^{1 / 2}\right)^{1 / 2}<r\right\} .
$$

The above theorem can be extended to $p$-harmonic functions (see Theorem 6.1.1 A]) or solutions of linear elliptic equations with real analytic coefficients ([DS] and [E]).

Theorem 3.2 alone is not enough to show equivalence of the holomorphic and harmonic cases since restriction of a locally integrable function to lower-dimensional hyper-planes can result in a locally non-integrable function. It seems worth investigating if restricting holomorphic functions to a fixed lower-dimensional surface gives rise to different stability results depending on the position of the surface. 


\section{ACKNOWLEDGments}

The author thanks Dima Khavinson for some valuable conversations. Thanks are also due to D. H. Phong for his lectures during the Workshop on Analysis and Resolution of Singularities at the CRM/ISM, Montréal 2003. The author acknowledges the support and stimulating atmosphere of CRM/ISM and CIRGET, and in particular V. Apostolov, G. Dafni and D. Jakobson, during his stay in Montréal.

\section{REFERENCES}

[A] M. F. Atiyah, Resolution of singularities and division of distributions, Comm. Pure Appl. Math. 23 (1970), 145-150 MR0256156 (41:815)

[Av] V. Avanissian, Cellule d'harmonicité et prolongement analytique complexe, Hermann, Paris, 1985 MR0851008 (87j:31009)

[AKS] N. Aronszajn, A. Krzywicki, J. Szarski, A unique continuation theorem for exterior differential forms on Riemannian manifolds, Arkiv för Matematik 4 (1962), 417-435 MR0140031 (25:3455)

[Be] I. N. Bernstein, Analytic continuation of generalized functions with respect to a parameter, Funkcional. Anal. i Priložen. 6 (1972), no. 4, 26-40 MR0320735 (47:9269)

[BG] I. N. Bernšteı̌n and S. I. Gel'fand, Meromorphy of the function $P^{\lambda}$, Funkcional. Anal. i Priložen. 3 (1969), no. 1 MR 0247457 (40:723)

[Bj] J.-E. Björk, Rings of differential operators, North-Holland, Amsterdam, 1979 MR 0549189 (82g:32013)

[BS] J.-M. Bony and P. Schapira, Existence et prolongement des solutions holomorphes des équations aux dérivées partielles, Invent. Math. 17 (1972), 95-105 MR0338541 (49:3305)

$[\mathrm{CF}] \quad$ R. R. Coifman and C. Fefferman, Weighted norm inequalities for maximal functions and singular integrals, Studia Math. 51 (1974), 241-250 MR0358205 (50:10670)

[CM] J. Cassaigne and V. Maillot, Hauteur des hypersurfaces et fonctions zêta d'Igusa, J. Number Theory 83 (2000), no. 2, 226-255 MR1772614 (2001k:11117)

[DK] J.-P. Demailly and J. Kollár, Semi-continuity of complex singularity exponents and KählerEinstein metrics on Fano orbifolds, Ann. Sci. École Norm. Sup. (4) 34 (2001), no. 4, 525-556 MR1852009(2002e:32032)

[DS] D. Khavinson and H. S. Shapiro, The Vekua hull of a plane domain, Complex Variables Theory Appl. 14 (1990), no. 1-4, 117-128 MR.1048711 (91e:35011)

[E] P. Ebenfelt, Holomorphic extension of solutions of elliptic partial differential equations and a complex Huygens' principle, J. London Math. Soc. (2) 55 (1997), no. 1, 87-104 MR 1423288 (98g:35029)

[GG] N. Garofalo and P. B. Garrett, $A_{p}$-weight properties of real analytic functions in $R^{n}$, Proc. Amer. Math. Soc. 96 (1986), no. 4, 636-642 MR0826494 (87g:42036)

[GL1] N. Garofalo, F. H. Lin, Monotonicity properties of variational integrals, $A_{p}$ weights and unique continuation, Indiana Univ. Math J. 35-2 (1986), 245-268 MR0833393|(88b:35059)

[GL2] Unique continuation for elliptic operators: a geometric-variational approach, Comm. Pure Appl. Math. 40 (1987), 347-366 MR0882069 (88j:35046)

[GV] N. Garofalo, D. Vassilev, Strong unique continuation of solutions of equations involving generalized Baouendi-Grushin operators, preprint 2003, http://comp.uark.edu/ dvassil/papers.html

[K] I. Kukavica, Quantitative uniqueness for second order elliptic operators, Duke Math. J. 91 (1998), no. 2, 225-240 MR.1600578 (99a:35046)

[Ka] M. Kashiwara, B-functions and holonomic systems. Rationality of roots of B-functions, Invent. Math. 38 (1976/77), no. 1, 33-53 MR0430304 (55:3309)

[M] B. Malgrange, Sur les polynômes de I. N. Bernstein, in Séminaire Goulaouic-Schwartz 1973-1974: Équations aux dérivées partielles et analyse fonctionnelle, Exp. No. 20, 10 pp, Centre de Math., École Polytech., Paris, 1974 MR0409883 (53:13635)

[N] A. M. Nadel, Multiplier ideal sheaves and Kähler-Einstein metrics of positive scalar curvature, Ann. of Math. (2) 132 (1990), no. 3, 549-596 MR.1078269 (92d:32038)

[PS1] D. H. Phong and J. Sturm, Algebraic estimates, stability of local zeta functions, and uniform estimates for distribution functions, Ann. of Math. (2) 152 (2000), no. 1, 277329 MR 1792297 (2002f:11180) 
[PS2] - On the algebraic constructibility of varieties of integrable rational functions on $C^{n}$, Math. Ann. 323 (2002), no. 3, 453-484 MR.1923693 (2004b:32011)

[PSS] D. H. Phong, E. M. Stein and J. A. Sturm, On the growth and stability of real-analytic functions, Amer. J. Math. 121 (1999), no. 3, 519-554 MR1738409(2002a:58025)

[RSt] F. Ricci and E. M. Stein, Harmonic analysis on nilpotent groups and singular integrals. I. Oscillatory integrals, J. Funct. Anal. 73 (1987), no. 1, 179-194 MR0890662 (88g:42023)

[St1] E. M. Stein, Oscillatory integrals in Fourier analysis, in Beijing lectures in harmonic analysis (Beijing, 1984), 307-355, Ann. of Math. Stud., 112, Princeton Univ. Press, Princeton, NJ, 1986 MR0864375 (88g:42022)

[St2] Harmonic analysis: real-variable methods, orthogonality, and oscillatory integrals, Princeton Mathematical Series 43, Princeton University Press MR.1232192 (95c:42002)

[V] A. N. Varčenko, Newton polyhedra and estimates of oscillatory integrals, Funkcional. Anal. i Priložen. 10 (1976), no. 3, 13-3 MR0422257 (54:10248)

CRM/ISM - UQAM, C.P. 8888, succursale Centre-Ville, Montréal, Québec, Canada H3C 3P8 - and - Mathematical Science Department, University of Arkansas, FayetTEVILle, Arkansas 72703

E-mail address: vassilev@math.uqam.ca 